\title{
FORMULATION OF DRIVER JUDGMENT PROCESS AROUND CURVES FOR DEVIATED STATE DETECTION
}

\author{
Motoki Shino $^{1}$, Hiroshi Yoshitake ${ }^{1}$, Machiko Hiramatsu ${ }^{2}$, \\ Takashi Sunda $^{2}$ \& Minoru Kamata ${ }^{1}$ \\ ${ }^{1}$ The University of Tokyo \\ ${ }^{2}$ Nissan Motor Co. Ltd. \\ Tokyo, Japan \\ Email: motoki@sl.t.u-tokyo.ac.jp
}

\begin{abstract}
Summary: The objective of this study is to propose indices that detect deviated states of drivers during driving considering their judgment process and using a road environment and natural driving behavior database. To this end, we focus on curved roads because as road curvature changes continuously drivers need to adapt their behavior to not go wide of the lane or the road. The drivers' speed choice behavior around curve situations was focused upon, and a speed choice process was formulated. Moreover, the validity of the formulated speed choice behavior in curve situations was examined using the real vehicle and the simulator.
\end{abstract}

\section{INTRODUCTION}

In Japan, the number of traffic accidents occurring annually remains high. A recent estimate puts the number at 700,000. To decrease this number, studies on active safety technologies including driver assistance systems are being carried out. Such systems can prevent accidents by checking any unsafe driving behavior by drivers. Typically, unsafe driving behavior is ascribed to the following:

1. The driver is an accident-prone person in general.

2. The driver is not an accident-prone person, but their driving behavior in a given situation is different compared to their normal driving behavior.

In this study, we focused on drivers with the second feature, and driving behavior different from their usual pattern is defined as "deviated" driving behavior. Individuals have unique driving behaviors, and it is assumed that driver state affects the driving behavior. Thus, the deviated state is defined as the state in which driver behavior and state are not normal. It is assumed that unsafe driving is part of deviated driving behavior. If drivers' deviated state is detectable and a driver assistance system that recovers driver state from deviated to normal is realized, we can prevent accidents resulting from unsafe driving behavior. We focus on detecting the deviated state based on driving behavior. When driving under various circumstances, we vary steering wheel and pedal operation based on the road environment. In this light, it is important to consider the road environment as well as driving behavior for detecting the driver state. Moreover, given that drivers' judgment process, through which a driver selects driving behavior based on the road environment, is influenced by the driver state, it is important to consider this process as well.

Figure 1 shows the schematic diagram of the proposed deviated state detection system considering the driver judgment process. The driving behavior database used here records not only driving behavior data but also road environment data. Using this database, we design an action-select model that takes road environment data as input and predicts suitable driving behavior data as output. Thus, driving behavior prediction is realized, and deviated driver state is detected by comparing the predicted and measured driving behaviors. To realize this detection 
method, we first need to materialize the action-select model that is related to the driver judgment process. Therefore, the objective of this paper is to examine and formulate the driver judgment process using the driving behavior database.

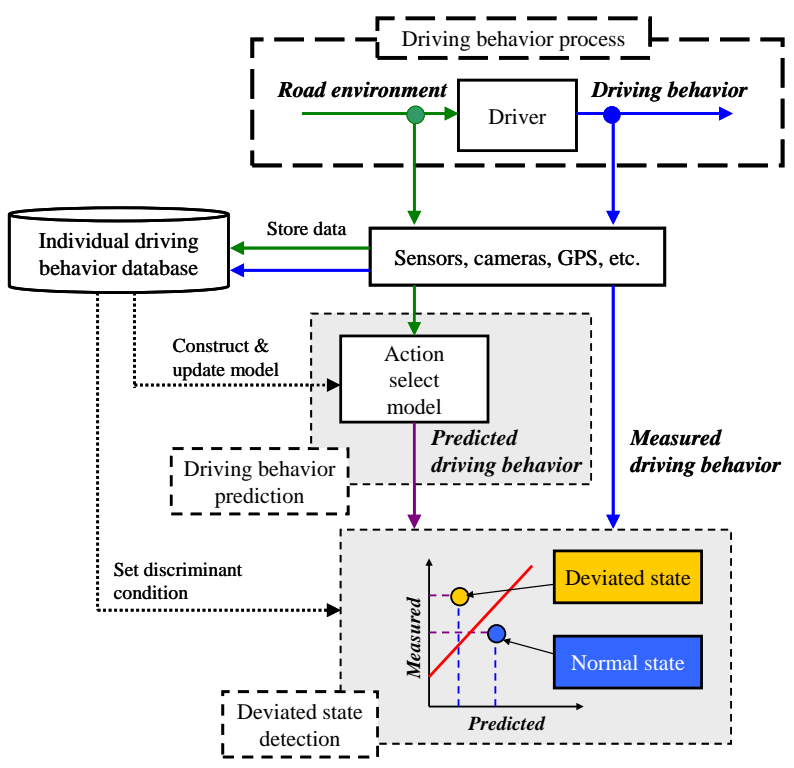

Figure 1. Schematic diagram of deviated state detection method

\section{DRIVING SITUATION AND DRIVING BEHAVIOR INDEX FOR DETECTING DEVIATED STATE}

\section{Driving Situation}

We decided to focus on a curved road as the driving situation. In this situation, road curvature changes continuously, and drivers need to adapt their behavior accordingly to ensure that their vehicle does not go wide of the lane or the road. As such, the choice of a suitable driving behavior is important in this situation. Therefore, it could be assumed that changes in driver state play a major role in any road-environment-based change in driving behavior. Thus, in this study, we focused on the curve situation for detecting deviated driving states. Although a curve situation can be defined based on road geometry from map data, here it is defined using driving behavior data.

\section{Driving Behavior Index for Detecting Deviated State}

In this study, we aim to detect deviated driver states from recorded driving behaviors by focusing on the judgment process relative to the road environment. To this end, a driving behavior index that satisfies the following two conditions is used for detecting deviated states.

1. Have relation with road environment

2. Indicate driver state

A previous study (Takahashi et al, 2010) shows that the velocity selected by a driver in a curve situation is related to the road curvature in said situation. In this light, we examined whether average vehicle velocity in a curve section $V_{a v g}$ satisfies the two abovementioned conditions, for selecting it as an index candidate. 


$$
V_{\text {avg }}=\frac{1}{L} \int_{0}^{L} V(l) d l
$$

where $V$ denotes vehicle velocity and $L$ denotes the curve situation distance. To examine whether the index satisfies these conditions, we used the Research Institute of Human Engineering of Quality Life (HQL)'s driving behavior database (HQL database), which contains driving behavior data of approximately 100 individuals (Akamatsu, 2003). We used the driving data of four drivers, IA, IB, IC, and ID, who drove through the same course on public roads. First, to examine the relationship with road curvature, a correlation coefficient between $V_{\text {avg }}$ and average radius of curvature $R_{a v g}$ was calculated. The calculation results (Table 1 ) show that there is adequate correlation between $V_{a v g}$ and $R_{a v g}$, thus indicating that the index $V_{a v g}$ has a specific relation with the road environment. Next, to verify whether $V_{\text {avg }}$ indicates driver state, we compared normal and deviated trips. These trips have been defined in a previous study (Yoshitake et al., 2011), and it is shown that these classified trip data correspond to the normal state and deviated state of drivers. Therefore, if $V_{\text {avg }}$ for normal trip data and deviated trip data is different, $V_{\text {avg }}$ can be said to correspond to driver state. Figure 2 shows the result of normal and deviated trip data comparison of $V_{a v g}$ for each driver. It can be seen that with the exception of driver IB, the $V_{a v g}$ values corresponding to deviated trip data are significantly higher than those corresponding to normal trip data. This indicates that the examined index is related to driver state. From the result of these examinations, we can say that $V_{\text {avg }}$ satisfies the two conditions required of an index for detecting deviated state. Therefore, $V_{\text {avg }}$ was adopted for detecting deviated driver states.

Table 1. Results of correlation coefficient t-test $\left(V_{\text {avg }} \& R_{\text {avg }}\right)$ based on curve group data

\begin{tabular}{|c|c|c|c|}
\hline Driver ID & $\begin{array}{c}\text { Correlation } \\
\text { coefficient } r\end{array}$ & $\begin{array}{c}\text { Curve data } \\
\text { frequency } N\end{array}$ & P value \\
\hline \hline IA & 0.738 & 329 & $7.32 \times 10^{-58}$ \\
\hline IB & 0.773 & 280 & $5.94 \times 10^{-57}$ \\
\hline IC & 0.742 & 382 & $7.03 \times 10^{-68}$ \\
\hline ID & 0.706 & 314 & $1.34 \times 10^{-48}$ \\
\hline
\end{tabular}

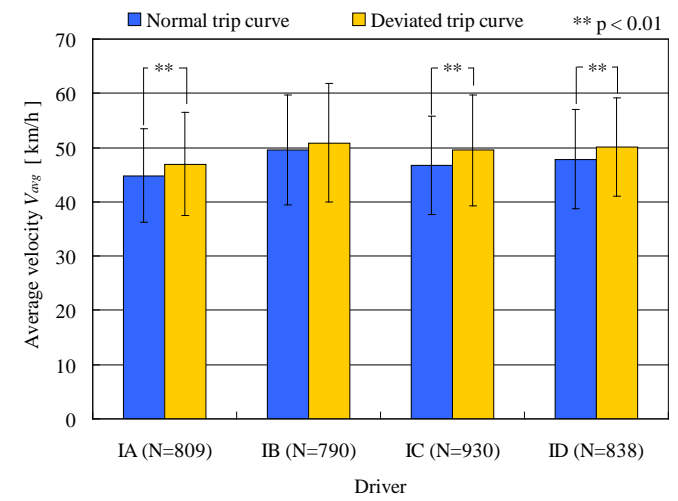

Figure 2. Result of two-sample t-test (Normal trip curve \& deviated trip curve)

\section{SPEED CHOICE BEHAVIOR HYPOTHESIS}

\section{Road Environments Affecting Speed Choice Behavior}

It is assumed that speed choice behavior in curve sections is affected by many road environment conditions such as road curvature, lane width, preceding vehicle, and oncoming vehicle. Although drivers change speed to decrease the possibility of exceeding the lane or road, road geometry features such as road curvature or lane width of the curve section tend to majorly influence drivers' choice of speed. Therefore, we focused on these two factors as road environments affecting speed choice. 


\section{Application of TLC Concept to Curve Driving}

According to the previous section, the speed choice behavior of drivers in curve driving can be described using two parameters: road curvature and lane width. To materialize this speed choice process, we applied the time-to-line-collision (TLC) concept, introduced by Godthelp (Godthelp et al., 1984), to curve driving. Figure 3 shows the result of this application, and Eq. 2 presents an index TLC that indicates drivers' speed choice in curve driving derived from the result shown in the figure.

$$
V=\frac{\left(1-k^{\prime}\right) R}{T L C} \cos ^{-1}\left(1-\frac{d(2 R-d)}{2 R^{2} k^{\prime}\left(1-k^{\prime}\right)}\right)
$$

where $R$ indicates the radius of curvature; $V$, the velocity; $2 \mathrm{~d}$, the lane width; and $k$, the steering error coefficient. Eq. 2 suggests that the speed selected in curve driving can be predicted based on $R$ and $d$. However, this equation can be applied as a speed prediction model only if the TLC is not related to $R$ and $d$. To examine the possibility of using Eq. 2 as a speed prediction model, we examined the relation between TLC and $R$ using the HQL database. In this study, as a representative index for the curvature of curved road sections, $R_{\text {avg }}$ was adopted. Furthermore, to exclude the effect of lane width, the curve data stored in the HQL database was classified based on lane condition. Table 2 summarizes lane condition classification of curve sections. In the HQL database, because lane condition groups other than LC1, LC2, and LC6 had insufficient curve data for examining the relationship between $T L C$ and $R_{a v g}$, we conducted said examination using the curve data of LC1, LC2, and LC6.

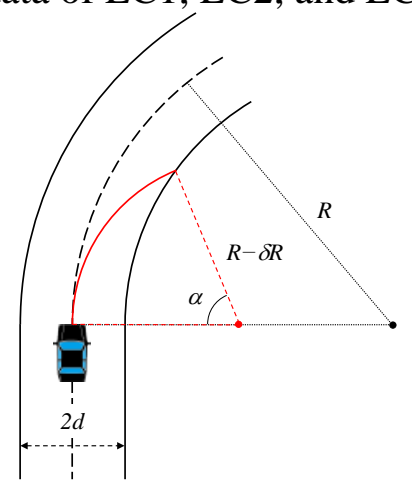

Table 2. Definition of lane condition group

\begin{tabular}{|c|c|c|c|c|c|c|c|c|c|}
\hline $\begin{array}{c}\text { Lane } \\
\text { condition } \\
\text { group }\end{array}$ & LC1 & LC2 & LC3 & LC4 & LC5 & LC6 & LC7 & LC8 & LC9 \\
\hline $\begin{array}{c}\text { Road } \\
\text { width } \\
\text { [m] }\end{array}$ & 4.0 & 4.5 & 5.5 & 6.0 & 6.5 & 5.5 & 6.0 & 6.5 & 7.0 \\
\hline $\begin{array}{c}\text { Existence } \\
\text { of } \\
\text { centerline }\end{array}$ & $\mathrm{x}$ & $\mathrm{x}$ & $\mathrm{x}$ & $\mathrm{x}$ & $\mathrm{x}$ & 0 & 0 & 0 & 0 \\
\hline
\end{tabular}

Figure 3. TLC concept diagram for curve

\section{TLC Examination Based on HQL Database}

The result of the $T L C-R_{\text {avg }}$ comparison threw up two major tendencies. Figure 4 shows sample data corresponding to these tendencies. In the regions where $R_{a v g}$ was small, there was no significant correlation between TLC and $R_{\text {avg }}$. However, in the regions where the value of $R_{a v g}$ was greater than $200 \mathrm{~m}$, the length of TLC increased with the $R_{a v g}$ value. This means that drivers decreased vehicle speed as $R_{\text {avg }}$ increased. To examine the cause of this tendency, we examined the relationship between $V_{a v g}$ and $R_{a v g}$. Figure 5 shows the result of a comparison between $V_{a v g}$ and $R_{a v g}$. The comparison indicates that the gradient of $V_{\text {avg }}$ decreases as $R_{\text {avg }}$ increases. This result suggests that the $V_{\text {avg }}$, or the driver-selected speed, is approaching some constant value. The larger the $R_{\text {avg }}$, the straighter is the road. Therefore, the constant value might be determined based on the velocity that drivers choose on straight roads; this could act as a permissible velocity value. 
From the results of examining the HQL database, we developed two hypotheses regarding speed choice behavior in curve driving. The first hypothesis is that drivers tend to maintain the TLC and adapt their driving speed in curves according to the road curvature. The second hypothesis is that when the road curvature increases, or the road become straighter, drivers tend to set their driving speed to a certain permissible velocity related to speeds selected on straight roads.

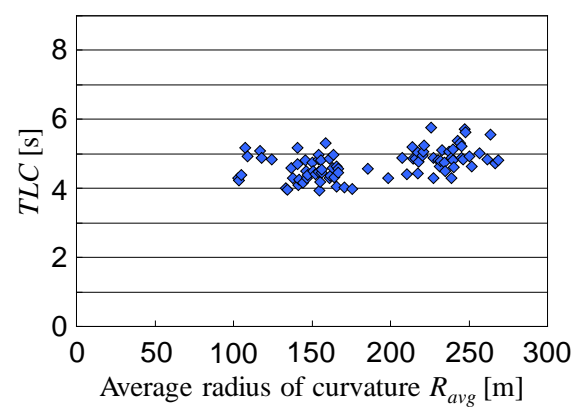

Figure 4. Relation between $T L C$ and $R_{a v g}$ (Subject IA, Lane condition: LC6)

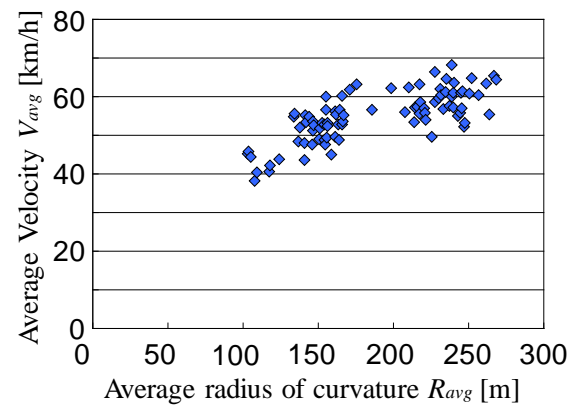

Figure 5. Relation between $V_{\text {avg }}$ and $\boldsymbol{R}_{\text {avg }}$ (Subject IA, Lane condition: LC6)

\section{VALIDATION OF SPEED CHOICE BEHAVIOR HYPOTHESES}

\section{Examination Conditions}

The speed choice behavior hypotheses have been developed based on the HQL database. However, in databases containing driving behavior data of various individuals, there is noise of some variety. Therefore, we examined the validity of these hypotheses in two different ways. First, curve data collected using a real vehicle and a test course were used for validating the hypotheses. The test course contained 8 curve sections of curvatures ranging from $50 \mathrm{~m}$ to 150 m. Second, curve data collected using a driving simulator and four drivers (DA, DB, DC, and $\mathrm{DD})$ were used to examine the hypotheses. The test course set on the driving simulator contained 8 curve sections with curvatures ranging from $50 \mathrm{~m}$ to $200 \mathrm{~m}$ and two different lane widths (2.75 $\mathrm{m}$ and $3.50 \mathrm{~m})$.

\section{Examination Result Based on Driving Simulator Curve Data}

Figure 6 shows a sample result (Driver DA) of the relationship between TLC and $R_{\text {avg. There is }}$ significant correlation between TLC and $R_{a v g}$ as $R_{a v g}$ increases. This finding suggests that drivers tend decreases speed as $R_{\text {avg }}$ increases. Figure 7 shows a sample result of the relationship between $V_{\text {avg }}$ and $R_{\text {avg }}$. Here, we considered $150 \mathrm{~m}$ as a set value of $R_{\text {avg }}$ and divided the curve data into two groups according to the set value. Upon comparing the $V_{a v g}-R_{a v g}$ correlation coefficients of the two groups, we found significant differences between the two groups. This indicates that drivers' the speed choice strategies changed according to $R_{a v g}$. In the curve data for $R_{\text {avg }}$ greater than $150 \mathrm{~m}$, there was no significant correlation between $V_{\text {avg }}$ and $R_{\text {avg. This suggests }}$ that the speed selected by drivers approaches a constant value and is based on individual permissible speeds.

From the results of an examination using driving simulator curve data, we verified the two hypotheses of speed choice behavior derived from database analysis. Therefore, it can be said that there are two types of speed choice behavior. In the first type, driving speed is selected based 
on road geometry, while in the second type, it is selected based on the permissible velocity in straight road driving. These two different types of speed choice in curve sections are formulated in the next chapter.

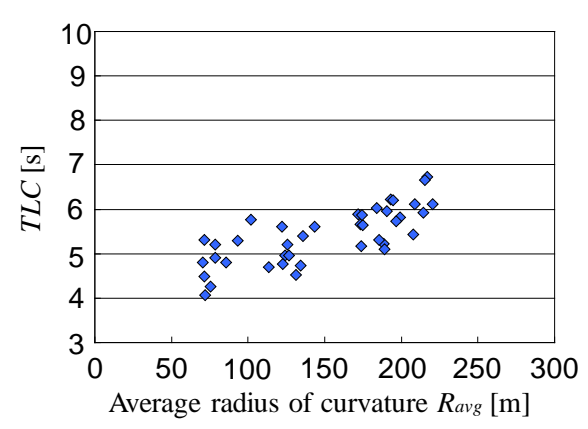

Figure 6. TLC vs. $R_{\text {avg }}$ (DA, $\left.2.75 \mathrm{~m}\right)$

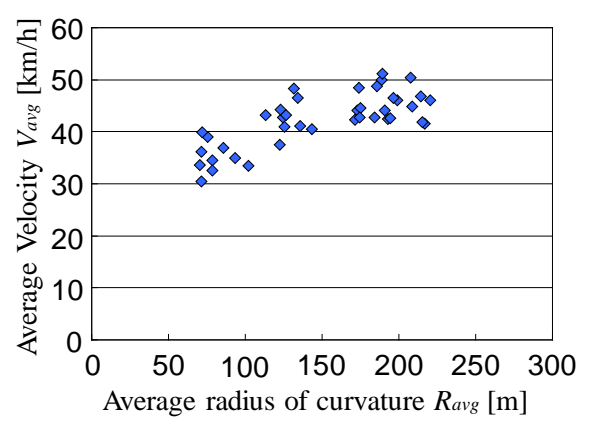

Figure 7. $V_{\text {avg }}$ vs. $R_{\text {avg }}(\mathrm{DA}, 2.75 \mathrm{~m})$

\section{FORMULATION OF SPEED CHOICE BEHAVIOR IN CURVE SITUATION}

\section{Model Equation}

The two types of speed choice are formulated based on their respective features. First, the speed choice type in which driving speed is selected according to road geometry is formulated using Eq. 2 because the TLC is constant regardless of road curvature. Next, the speed choice type in which driving speed is selected according to permissible velocity is formulated using exponential functions because the speed chosen in curve driving approaches a constant value as the road curvature increases (Eq. 3).

$$
V=\alpha\left(1-\exp \left(-\left(R-R_{b}\right) / \beta\right)\right)+V_{b}
$$

where $R_{b}$ is a boundary value between Eqs. 2 and 3, and the value is determined from the characteristics that there is no significant correlation between $R_{a v g}$ and $V_{a v g}$ which are obtained the driving behavior data in curve situation. $V_{b}$ is the average speed at $R_{b} . \alpha$ and $\beta$ are identified to minimize the prediction error between the value obtained using Eq. 3 and the driving behavior data in curve situation where $R_{\text {avg }}$ was large over $R_{b}$. Figure 8 shows the speed choice model obtained using Eqs. 2 and 3 and the driving behavior data for curve sections. It can be confirmed the model of curve driving is expressible.

\section{Evaluation of Formulated Model Equation}

To verify the effectiveness of the formulated speed choice model, we calculate the prediction error using both Eqs. 2 and $3\left(\mathrm{PE}_{1}\right)$ and the predicted error using Eq. 2 only $\left(\mathrm{PE}_{2}\right)$. Figure 9 shows the speed choice model developed using only Eq. 2 as "TLC only" and the speed choice model developed using both Eqs. 2 and 3 as "Hybrid." Table 3 summarizes the results of prediction error with speed choice model by using the HQL database. It can be seen that for all four drivers, the Hybrid speed choice model has lower prediction error than the TLC only model. Therefore, the validity of the speed choice model in curve driving, which involves selection of driving speed based on road geometry and permissible velocity, was confirmed. 


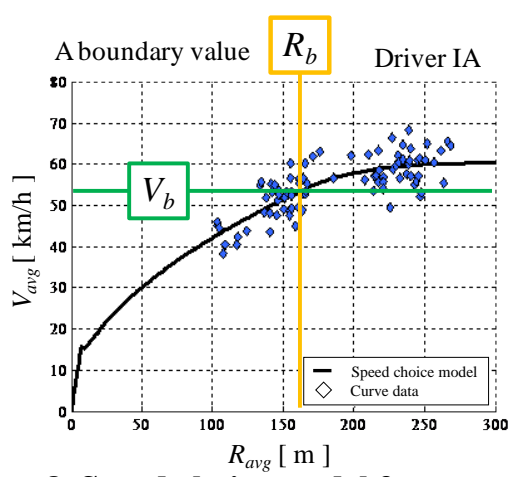

Table 3. Prediction error of each sneed choice model

Figure 8. Speed choice model for curve driving

\begin{tabular}{|c|c|c|c|}
\hline Driver ID & $\begin{array}{c}\text { Lane } \\
\text { condition } \\
\text { group }\end{array}$ & $\mathrm{PE}_{1}[\mathrm{~km}]$ & $\mathrm{PE}_{2}[\mathrm{~km}]$ \\
\hline \hline & LC1 & - & 4.64 \\
\hline IA & LC2 & - & 4.61 \\
\hline & LC6 & 4.03 & 4.73 \\
\hline & LC1 & 3.95 & 4.91 \\
\hline IB & LC2 & - & 3.89 \\
\hline & LC6 & 5.04 & 5.91 \\
\hline & LC1 & 4.47 & 4.62 \\
IC & LC2 & - & 5.34 \\
\hline & LC6 & 5.39 & 6.14 \\
\hline & LC1 & 5.54 & 5.7 \\
\hline ID & LC2 & - & 4.89 \\
\hline & LC6 & 5.49 & 6.57 \\
\hline
\end{tabular}

(Subject IA, Lane condition: LC6)

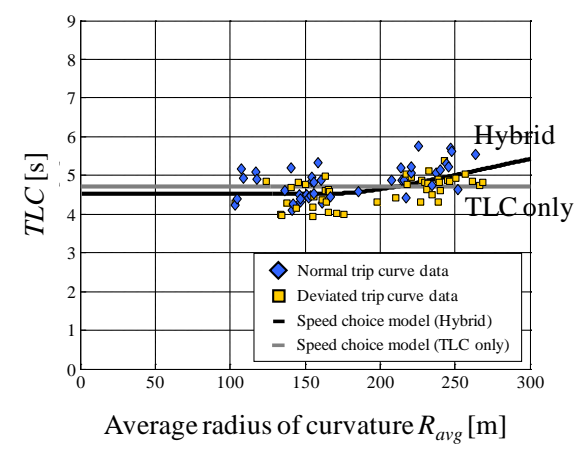

(a) $T L C$ vs. $R_{\text {avg }}$

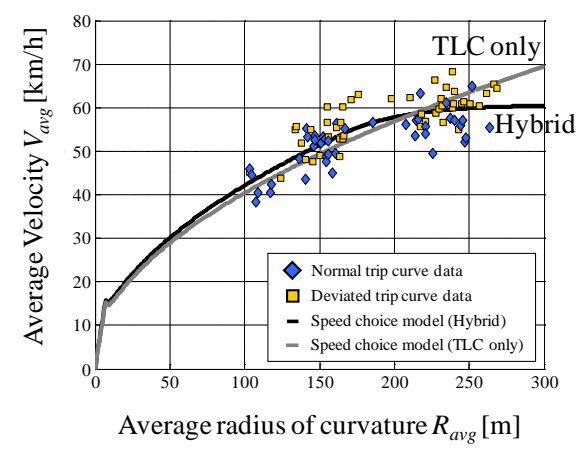

(b) $V_{a v g}$ VS. $R_{a v g}$

Figure 9. Comparison of speed choice models and database in curve driving

\section{CONCLUSIONS}

(Subject IA, Lane condition: LC6)

In this paper, we proposed a speed choice model for curve driving to detect deviated driver states and validated the proposed model using naturalistic driving behavior data. The major conclusions of this study are as follows:

- We can use average velocity as an index based on the formulation of speed choice behavior in curve driving.

- The formulation of speed choice behavior in curve driving, which consists of the driving speed according to road geometry and the permissible velocity, was proposed and validated.

\section{REFERENCES}

Akamatsu, M.. (2003). Establishing Driving Behavior Database and its Application to Active Safety Technologies, Journal of Society of Automotive Engineers of Japan, Vol.57, No.12, pp.34-39 (in Japanese).

Godthelp, H., et al. (1984). The Development of a Time-Related Measure to Describe Driving Strategy. Human Factors, Vol.26, No.3, pp.257-268.

Takahashi, A., et al. (2010). Speed Choice Model of Curve Entering Based on Driving Behavior Database. Transactions of Society of Automotive Engineering of Japan, Vol.41, No.5, pp.981986 (in Japanese).

Yoshitake, H., et al. (2011). Driver State Detection Method of Hurry State Based on Naturalistic Driving Behavior Database, Proc. of FAST-zero'11(CD-ROM), TS2-6-2-3. 